\title{
Hardware in the Loop Test-bed for Space Information Network
}

\author{
Chengguang Fan a, Lei Yang ${ }^{b}$, Yong Zhao c ${ }^{c}$, Shuai Wu ${ }^{d}$ and Jianming Guo ${ }^{e}$ \\ College of Aerospace Science and Engineering, National University of Defense Technology, \\ Changsha 410073, China \\ achengguangfannudt@163.com, bcraftyang@163.com, cfcggfs@126.com, \\ dwushuai163009@163.com, egjm08110@hotmail.com
}

Keywords: Test-Bed, Space Information Network, Hardware in the Loop, Simulation.

\begin{abstract}
In this paper, in the light of the diversity of space-crafts in space information network and the fast switching characteristics of network nodes, a hardware in the loop (HITL) test-bed is designed and built for testing and evaluating the appointed space information network, which consists of the modeling tool library, model library, scenario generation library, simulation driven module, data management and analysis module, simulation results visualization module, hardware equipment, as well as HITL interface. The detailed implement process of the test-bed is described for a given task, and the process and related result can be visually monitored. It is shown that the test-bed is necessary for space information network and can strongly support the demonstration and verification of the key techniques.
\end{abstract}

\section{Introduction}

In recent years, the space information network has been widely studied due to the fierce competition in space [1-3]. The network is based on the space platforms, which include satellite with different function running on various orbit altitudes, stratospheric balloon and aircraft. And it can achieve real-time space information acquisition, transport, as well as processing. As the most important national infrastructure, one side, it can be widely used in the area of ocean sailing, emergency rescue, navigation positioning, air transportation and aerospace measurement and control. On the other side, the space information network is a giant and complex system, involving various disciplines, the construct of which cannot be directly finished via theoretical calculation, thus experimental demonstrations are necessary. The simulated test-bed is a good choice before building the real space information network [4-5].

The test-bed has been widely used in the area of future internet design. So far there are some typical future internet test-beds in the world, such as the GENI [6] (Global Environment for Networking Innovations) in USA, the FIRE [7] (Future Internet Research and Experimentation) in Europe, the JGN-X [8] (Japan New Generation Network Test-bed) in Japan. In China, a test-bed termed sea-cloud platform aiming to next generation of information technology for sensing China has been proposed by Chinese Academy of Sciences [9]. It should be noted that all these mentioned test-beds are built to do the research on future ground internet, which is different from the space information network. Therefore, it is valuable to build the related test-bed for space information network. In this paper, the hardware in the loop (HITL) test-bed is studied to achieve the ground test of space information network.

\section{Test-bed Design and Description}

\subsection{Test-bed Design.}

This section designs the structure of the hardware in the loop test-bed for space information network, which includes the modeling tool library, model library, scenario generation library, simulation driven module, data management and analysis module, simulation results visualization module, hardware equipment, as well as HITL interface. The structure schematic diagram is shown in Fig.1. The detailed description for each component is followed. 


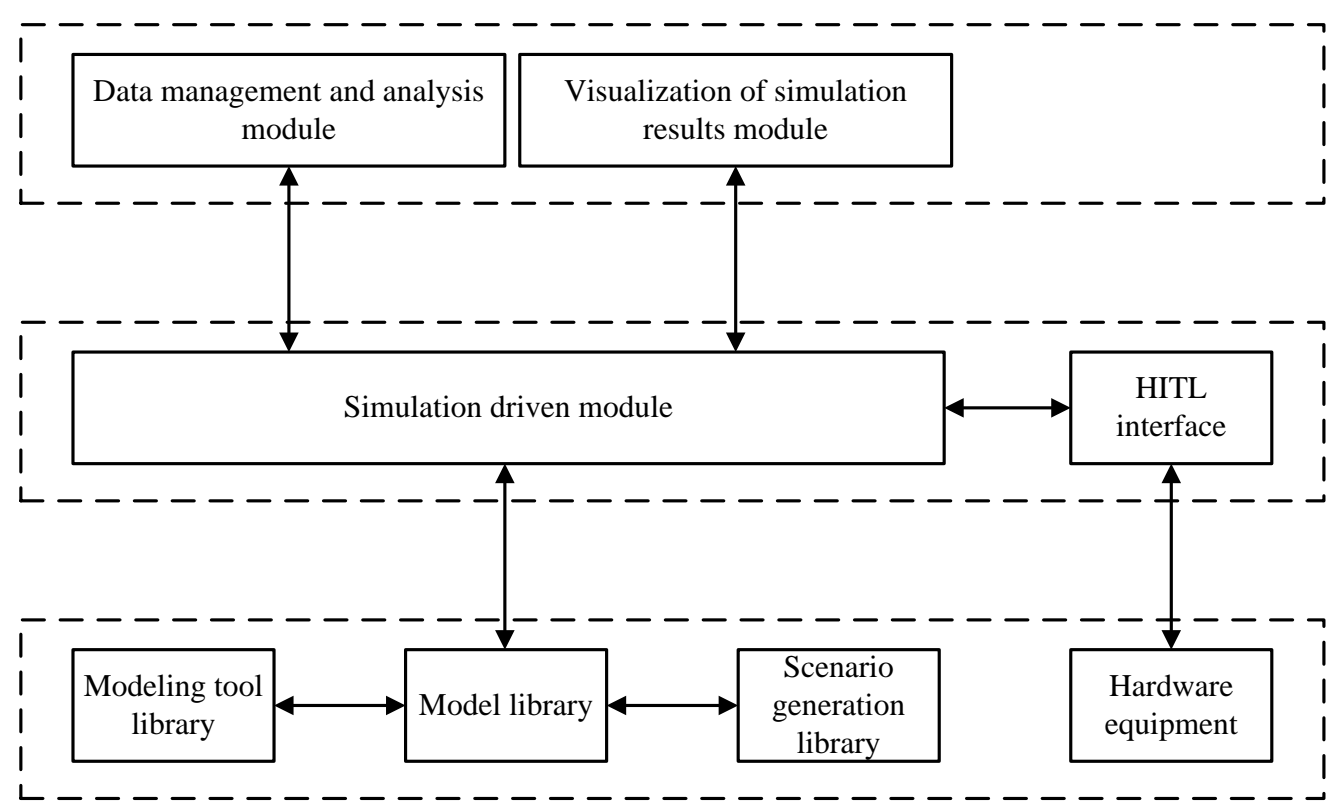

Fig. 1 The structure schematic diagram of the hardware in the loop test-bed

\subsection{Subsystem Description.}

Modeling tool library. The space information network is a huge system and involves different disciplines, such as orbit dynamics, attitude dynamics, wireless communication, as well as network science. In each discipline, the relatively mature modeling tool has been built, including OPNET, Qualnet, STK, NS2 et al. However, until now there is no one which can be used to simulate the whole network. Therefore, a modeling tool library including different tools is constructed. Based on these tools, an integrated simulation platform can be constructed. Fig. 2 shows the interface of the OPNET, a common modeling tool.

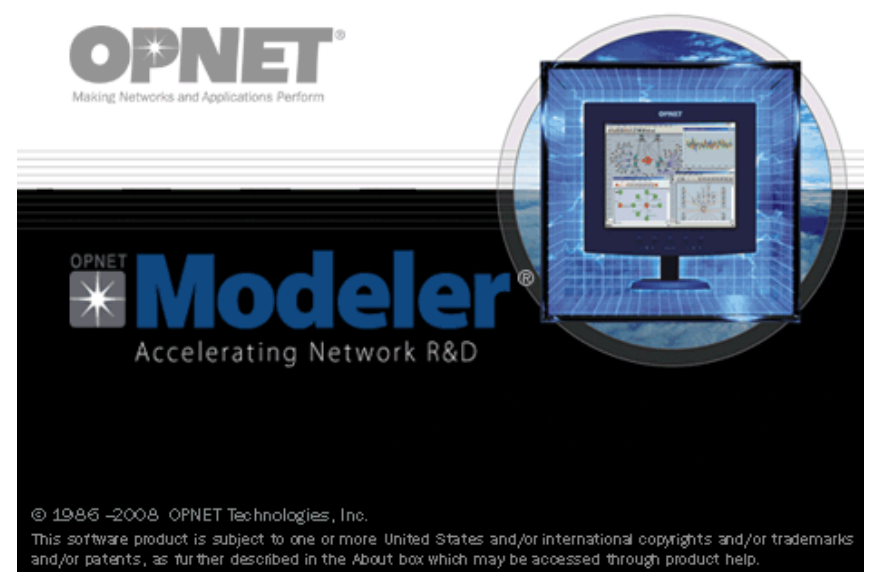

Fig. 2 The interface of the OPNET

Model library. The model library is built to provide and manage the model that can be used in the test-bed, such as satellite constellation model, orbit dynamics model, attitude dynamics model, wireless channel model, as well as network protocol model.

Scenario generation library. Various scenarios related with different application and service are built and saved in the scenario generation library. According to the assigned task, a related scenario is setup based on the constellation configuration, satellite structure and capability, network task requirement. Fig. 3 shows a scenario for space self-organizing network.

Simulation driven module. The space information network includes all kinds of space-craft, which has different structure, function and usage, resulting in different system mechanism and external interface. Therefore, the network is heterogeneous, and the generalized simulation driven is needed to drive different model. In addition, the distributed parallel computing is chosen to improve simulation speed. 


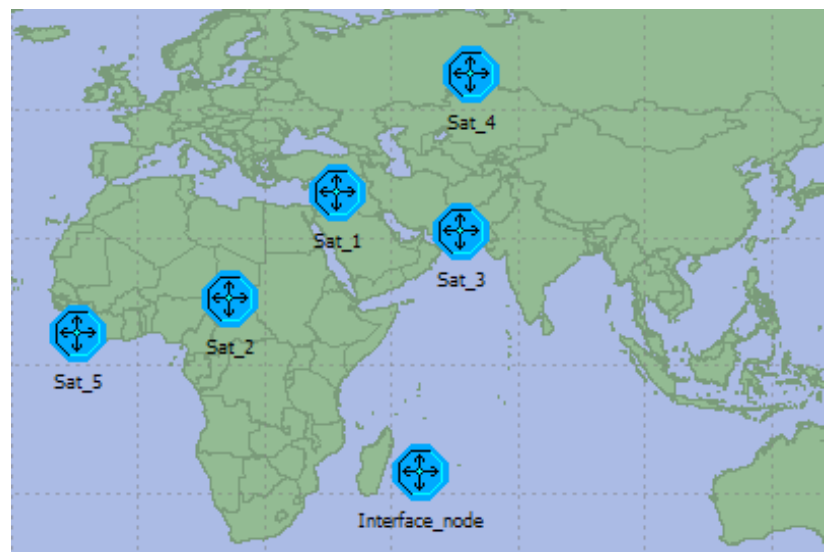

Fig. 3 The scenario setup for self-organizing network

Data management and analysis module. There are mass simulated and actual data to be processed in the test-bed. It is necessary to finish the data capture, storage and classification automatically. By analyzing these data the functional testing and performance evaluation of assigned space information network can be achieved.

Visualization of simulation results module. The output of hardware in the loop test-bed can be set in the form of two dimension display, three dimension display, also figure and table according to different output requirements. In addition, the whole process and related result can be visual tracked and displayed.

Hardware equipment and HITL interface. The test-bed also includes some hardware equipment, such as router, switch, radio station, transceiver, as well as simulated satellite. The hardware used in the test-bed is connected with the simulation part via HITL interface. Fig. 4 shows the physical figure of a simulated satellite used in the test-bed.

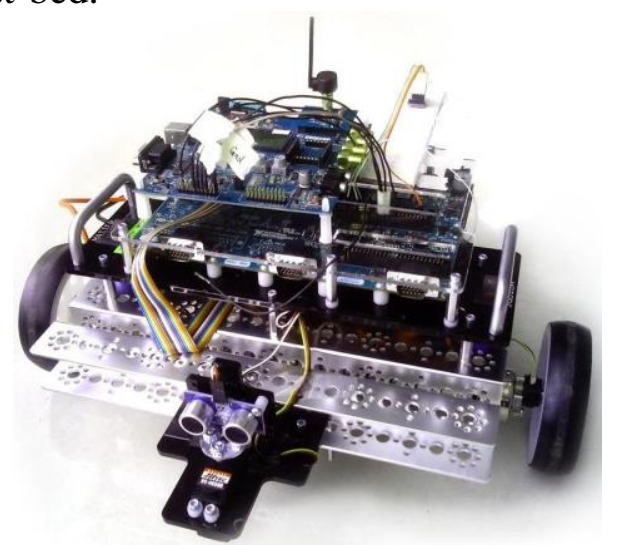

Fig. 4 The simulated satellite

\section{The Implement of Test-bed}

The second part of this paper has shown the structure of the hardware in the loop test-bed for space information network, and this part shows how the test-bed can be implemented for a given simulation task. The detailed process is shown in Fig.5.

Firstly, the simulation scenario based on the given task is analyzed and chosen from the scenario generation library.

Secondly, according to the generated scenario, the modeling tool, model and hardware are chosen from different library. The whole test-bed can be established via these components. And a proper HITL interface is chosen to connect the hardware with other parts.

Thirdly, the simulation driven is studied to ensure the implement of whole hardware in the loop test-bed successfully, which is the most important stage in the whole simulation.

Finally, the data obtained from the test-bed is captured and analyzed. The visualized result is exported and provides the conclusion of the given task. 


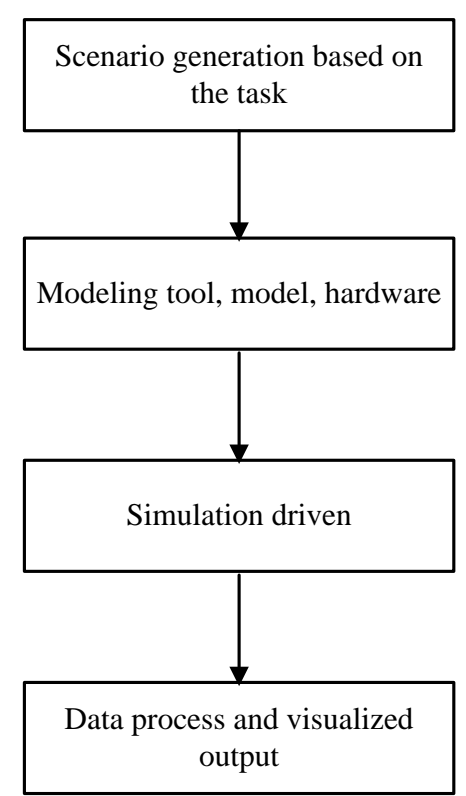

Fig. 5 The implement process of test-bed

\section{Summary}

This paper has described an investigation into the hardware in the loop test-bed for space information network. The structure of test-bed is given and each component is detailed explained. For a given task, a related scenario is designed firstly. And then the modeling tool, model as well as hardware are chosen to establish the test-bed, in which the HITL interface is selected to connect different parts. After that the simulation driven is studied to make sure the smooth running of the whole test-bed. Finally, the data obtained from the test-bed is processed and the result is displayed with different forms. The establishment of the test-bed can provide the technique support for space information network.

\section{References}

[1]. Zhou Hongbin, Yin Bo. Research on spatial information network system validation technology. Radio Communications Technology. Vol. 42 (2016) No. 2, p. 16-19, 47.

[2]. Li Deren, Shen Xin, Gong Jianya, et al. On construction of China's space information network. Geomatics and Information Science of Wuhan University. Vol. 40 (2015) No. 6, p. 711-715, 766.

[3]. Zhao Naitong, Zhao Kanglian, Liu Gongliang. Thought on constructing the integrated space-terrestrial information network. Journal of CAEIT. Vol. 10 (2015) No. 3, p. 223-230.

[4]. Liang Junxue, Lin Zhaowen, Ma Yan. Future internet experiment platform. Chinese Journal of Computers. Vol. 36 (2013) No. 7, p. 1364-1374.

[5]. Sun Yuanze, Zhao Hongli, Yang Haitao, et al. Summary of network testbed research. Journal of Equipment Academy. Vol. 25 (2014) No. 5, p. 88-92.

[6]. Information on: www.geni.net

[7]. Information on: www.ict-fire.eu

[8]. Information on: www.jgn.nict.go.jp

[9]. Tian Jing, Wang Jinlin. Next generation of information technology for sensing China: cooperation of cloud and sea. Science China. Vol. 45 (2015) No. 10, p. 1229-1236. 